\title{
AN INFINITE GRAPH OF GIRTH 12
}

BY

\author{
ASIA IVIC WEISS
}

\begin{abstract}
From the regular hyperbolic honeycomb $\{3,6,3\}$ we derive regular honeycombs with finite numbers of toroidal cells. Joining centers of faces of these honeycombs to the midpoints of its edges we obtain trivalent symmetrical graphs. We investigate the relation between these honeycombs, their groups and the graphs embedded in them.
\end{abstract}

1. Introduction. Coxeter and Whitrow [1950, p. 429] showed that the points whose four coordinates are proportional to the integral solutions of the Diophantine equation $t^{2}-x^{2}-y^{2}-z^{2}=1$ are vertices of the hyperbolic honeycomb $\{4,4,3\}$. Coxeter suggested that it would be useful to find coordinates for all regular hyperbolic honeycombs as integral solutions of Diophantine equations since as a by-product we would obtain a simple representation for generators of the symmetry groups of these honeycombs. Garner in his Ph.D. Thesis [1964] discovered coordinates for two more hyperbolic honeycombs $\{6,3,4\}$ and $\{6,3,3\}$. In $\S 3$ we complete these investigations by giving coordinates for all cristallographic regular hyperbolic honeycombs as well as their truncations.

This is then used in $\$ 5$ to find the group whose Cayley diagram is an infinite trivalent symmetrical graph of girth 12. At the Conference on Graph Theory and Combinatorial Analysis held at the University of Waterloo, R. M. Foster [1966] presented a paper which included a table of 176 trivalent symmetrical graphs. Since then there has been a search to find more. A few more of these graphs have been found by Coxeter, Frucht, Harries, and by Foster himself. In $\S 6$ we describe two new trivalent symmetrical graphs with 486 and 1280 vertices. We also describe some of the known ones in a new and essentially different way.

A regular honeycomb is a symmetrical subdivision of a 3-dimensional manifold into a number of regular polyhedral cells, all alike. According to the usual definition each cell is a topological disc or ball. Grünbaum [1976] suggested that it would be of interest to consider honeycombs whose cells are of other topological types. In $\$ \S 6,7$ and 8 we use the results of $\S 3$ to construct a number of regular honeycombs with toroidal cells. Two of the most interesting are ${ }_{h}\{3,6,3\}_{h}, h=4$ or 6 , which have 5 and 27 cells, respectively, of type $\{3,6\}_{h}$. It may be conjectured that these are the only two "naturally generated" self-dual finite honeycombs with cells of type

Received by the editors December 22, 1982.

1980 Mathematics Subject Classification. Primary 51M20; Secondary 05C25, 52A45.

(1) 1984 American Mathematical Society $0002-9947 / 84 \$ 1.00+\$ .25$ per page 
$\{3,6\}_{h}$. Here "naturally generated" means that the honeycomb is built, step by step, from disjoint copies of $\{3,6\}_{h}$, identifying elements only as directed by the vertex figures $\{3,6\}_{h}$.

2. Regular hyperbolic honeycombs and their orthoschemes. A 3-dimensional honeycomb is a set of polyhedra, called cells, fitting together to fill all space just once, so that every face of each polyhedron belongs to exactly one other polyhedron. A honeycomb is said to be regular if its cells are regular and congruent. We use the Schläfli symbol $\{p, q\}$ to denote a regular polyhedron whose faces are regular $p$-gons, $q$ of which meet at any vertex. A regular honeycomb whose cells are $\{p, q\}$ 's, $r$ round each edge, is denoted by $\{p, q, r\}$. The arrangement of cells around a common vertex corresponds to the arrangement of faces of a polyhedron $\{q, r\}$ called the vertex figure.

In hyperbolic space $\mathbf{H}^{3}$ the dihedral angle of $\{p, q\}$ is less than that of the corresponding Euclidean polytopes and it can take value $2 \pi / r$ whenever $p, q, r$ are integers satisfying

$$
\sin \pi / p \cdot \sin \pi / r<\cos \pi / q
$$

with $(p-2)(q-2)<4$ and $(q-2)(r-2)<4$ (Sommerville [1929, p. 168]). The actual instances are $\{3,5,3\},\{4,3,5\},\{5,3,4\}$ and $\{5,3,5\}$. These honeycombs have finite cells and finite vertex figures. Making an extension (Coxeter [1968, pp. 199-214]) we may allow the dihedral angle of $\{p, q\}$ to take its minimum value $(1-2 / q) \pi$, so that the edges are infinitely long and the vertices are on the absolute. In this case the edges at a vertex are all parallel, and the vertex figure, being a tessellated horosphere, is isometric to the Euclidean tessellation $\{q, r\}$, where $(q-2)(r-2)=4$. These further honeycombs are $\{3,4,4\},\{3,3,6\},\{4,3,6\}$ and $\{5,3,6\}$. Their reciprocals $\{4,4,3\},\{6,3,3\},\{6,3,4\}$ and $\{6,3,5\}$ have proper vertices, but the centers of their cells are points on the absolute: each cell is inscribed in a horosphere. We also allow self-reciprocal honeycombs $\{6,3,6\},\{3,6,3\}$ and $\{4,4,4\}$ for which $(p-2)(q-2)=4$ and $(q-2)(r-2)=4$. Their vertices, as well as centers of cells, lie on the absolute.

With each honeycomb we can associate a characteristic orthoscheme $P_{1} P_{2} P_{3} P_{4}$ whose faces are planes of symmetry of the honeycomb (Coxeter [1974, p. 34]). We use the "Coxeter graph" (Bourbaki [1968, p. 194])

$$
\vec{p} q \vec{q} r
$$

to denote an orthoscheme associated with a honeycomb $\{p, q, r\}$. Let $R_{i}$ be the reflection in the faces of the orthoscheme which does not contain $P_{i}$. Then four reflections $R_{i}$ generate the symmetry group $[p, q, r]$ of $\{p, q, r\}$ with the presentation

$$
R_{i}^{2}=\left(R_{1} R_{2}\right)^{p}=\left(R_{2} R_{3}\right)^{q}=\left(R_{3} R_{4}\right)^{r}=1, \quad R_{3} \rightleftarrows R_{1} \rightleftarrows R_{4} \rightleftarrows R_{2}
$$

(Coxeter [1973, p. 188]).

The vertices of some hyperbolic orthoschemes can be conveniently described in terms of projective coordinates $x, y, z, t$ with an absolute quadric $\Omega=0$, where $\Omega$ is 


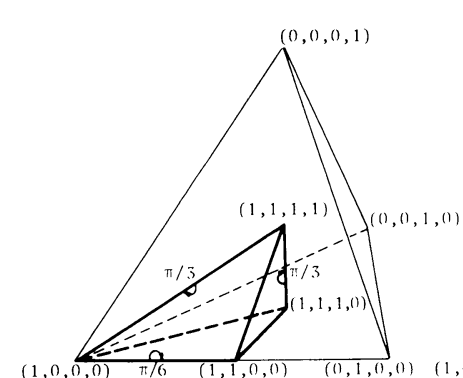

(a)

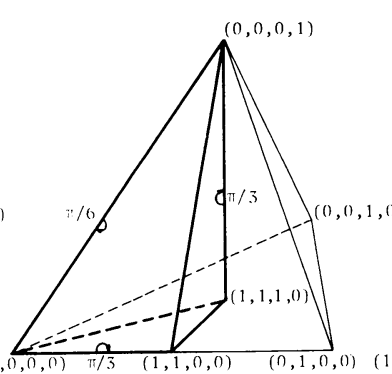

(b)

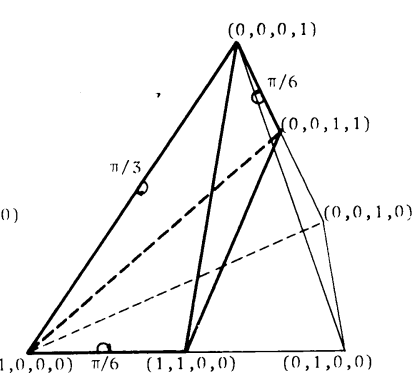

(c)

FIGURE 1

a suitable indefinite quaternary quadratic form. For this form let us first use

$$
\begin{aligned}
& \Omega_{1}=x y+x z+x t+y z+y t+z t \\
& =\left\{9(x+y+z+t)^{2}-6(x-y)^{2}-2(x+y-2 z)^{2}-(x+y+z-3 t)^{2}\right\} / 24 .
\end{aligned}
$$

Among many different tetrahedra inscribed in the absolute, we choose one whose dihedral angles are all $\pi / 3$, so as to obtain a regular tetrahedron $\{3,3\}$. For example $(1,0,0,0)$ together with all the permutations of its coordinates is such a tetrahedron (Figure 1). Points $(1,0,0,0),(1,1,0,0),(1,1,1,0)$ and $(1,1,1,1)$ are vertices of an orthoscheme

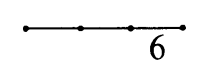

yielding regular honeycombs $\{3,3,6\}$ and $\{6,3,3\}$. With any regular tetrahedron inscribed in the absolute we can associate two other orthoschemes (Coxeter [1968, pp. 19-20]) like (b) and (c) in Figure 1, yielding self-reciprocal honeycombs $\{3,6,3\}$ and $\{6,3,6\}$, respectively.

With a regular hexahedron $\{4,3\}$ we can associate an orthoscheme for which a more convenient $\Omega$ is $\Omega_{2}=-3\left(x^{2}+y^{2}+z^{2}\right)+t^{2}$. Points $( \pm 1, \pm 1, \pm 1,3)$ are the vertices of a hexahedron inscribed in the absolute.

For another orthoscheme a more suitable $\Omega$ is $\Omega_{3}=-\left(x^{2}+y^{2}+z^{2}\right)+t^{2}$. The six points $( \pm 1,0,0,1),(0, \pm 1,0,1)$ and $(0,0, \pm 1,1)$, are the vertices of a regular octahedron $\{3,4\}$ inscribed in the absolute. With it we associate two orthoschemes:

$$
(0,1,0,1)(0,1,1,2)(1,1,1,3)(0,0,0,1)
$$

yielding $\{3,4,4\}$ and

$$
(0,1,0,1)(0,1,1,2)(0,0,0,1)(1,0,0,1)
$$

yielding $\{4,4,4\}$.

3. Coordinates for crystallographic hyperbolic honeycombs. The reflection in the plane $\rho(x, y, z, t)=0$ is the harmonic homology with axial plane $\rho(x, y, z, t)=0$ and center the pole of this plane with respect to $\Omega=0$. The faces of the orthoscheme 
opposite to $P_{1}, P_{2}, P_{3}$ and $P_{4}$ (in the coordinates described above) are in the planes $x=y, y=z, z=0$ and $t=0$, respectively. The four generating reflections $R_{i}$, $i=1,2,3,4$, for $[3,6,3]$ are as follows:

$R_{1}$ is the transposition $(x, y)$,

$R_{2}$ is the transposition $(y, z)$,

$R_{3}$ maps $(x, y, z, t)$ to $(x+z, y+z,-z, t+z)$,

$R_{4}$ maps $(x, y, z, t)$ to $(x+t, y+t, z+t,-t)$.

We will show that the vertices of $\{3,6,3\}$ are represented by the integral solutions of $\Omega_{1}=0$ and $x \equiv y \equiv z(\bmod 3)$ such that the greatest common divisor of $x, y, z$ and $t$ is 1 :

$$
\operatorname{gcd}(x, y, z, t)=1
$$

Clearly the orbit of the point $(0,0,0,1)$ will satisfy $\Omega_{1}=0$ since $(0,0,0,1)$ does and the $R_{i}$ are automorphs of the quadratic form $\Omega_{1}$. Also $R_{i}(i=1,2,3,4)$ does not change the following two conditions imposed on coordinates of a point: $x \equiv y \equiv z$ $(\bmod 3)$ and $\operatorname{gcd}(x, y, z, t)=1$. It remains to show that if $(x, y, z, t)$ is any integral solution of the three equations

$$
\Omega_{1}=0, \quad x \equiv y \equiv z(\bmod 3), \quad \operatorname{gcd}(x, y, z, t)=1,
$$

then, using transformations $R_{i}$, we can transform it to $(0,0,0,1)$.

If none of the coordinates $x, y, z$ and $t$ satisfying $\Omega_{1}=0$ is negative, then since $\operatorname{gcd}(x, y, z, t)=1$ the only possibilities are $(1,0,0,0)$ and any permutation of these coordinates. But $x \equiv y \equiv z(\bmod 3)$ rules out all permutations except $(0,0,0,1)$. Hence unless the point is $(0,0,0,1)$ there is at least one negative coordinate. We can assume $x+y+z+t \geqslant 0$ (otherwise take the "negative" quadruple). Now if $t<0$, apply $R_{4}$ to the point to get $(x+t, y+t, z+t,-t)$. The sum $x+y+z+2 t$ of its coordinates is positive, since $x+y+z+2 t<0$ would imply $x+y+z+t<-t$ and hence $x^{2}+y^{2}+z^{2}+t^{2}<t^{2}$ which is impossible. Furthermore if $t<0, x+y$ $+z+t<x+y+z+2 t$. Hence $R_{4}$ decreases the sum of the coordinates while leaving it positive. Since $R_{4}$ cannot decrease the sum indefinitely, if $t<0$ it must happen that eventually all the coordinates will be positive or zero. Since the remaining coordinates $x, y, z$ are permuted by $R_{1}$ and $R_{2}$, if one of them is negative we can assume $z<0$ and use $R_{3}$ to decrease the sum of all the coordinates. Eventually it must happen that all coordinates are $\geqslant 0$. This proves the above statement: $(x, y, z, t)$ can be transformed to $(0,0,0,1)$.

Coordinates for the rest of the crystallographic hyperbolic regular honeycombs, and their truncations, can be found in a similar way. The results are summarized in the tables below. Coordinates for the vertices of

$$
\{4,4,3\} \text { and }\left\{\begin{array}{r}
4 \\
4,3
\end{array}\right\}
$$


are already known, with respect to a different quadratic form (Coxeter and Whitrow [1950, pp. 428-429]), as well as coordinates for the vertices of $\{6,3,4\}$ (Garner [1964, p. 48]).

TABLE 1. Honeycombs using absolute $\Omega_{1}=0$, where $\Omega_{1}=x t+y t+z t+y z+z x+x y$.

\begin{tabular}{|c|c|}
\hline Honeycomb & Equations and congruences \\
\hline$\{3,3,6\}$ & $\Omega_{1}=0$ \\
\hline$\left\{\begin{array}{l}3 \\
3,6\end{array}\right\}$ & $\Omega_{1}=1$ \\
\hline$\{6,3,3\}$ & $\Omega_{1}=6, x \equiv y \equiv z \equiv t(\bmod 3)$ \\
\hline$\left\{\begin{array}{l}6 \\
3,3\end{array}\right\}$ & $\begin{array}{c}\Omega_{1}=3, x \equiv y \equiv z \equiv t+1(\bmod 3) \\
\text { for some permutation of } x, y, z \text { and } t\end{array}$ \\
\hline$\{3,6,3\}$ & $\begin{aligned} \Omega_{1}= & 0, x \equiv y \equiv z \equiv t(\bmod 3) \\
& \operatorname{gcd}(x, y, z, t)=1\end{aligned}$ \\
\hline$\left\{\begin{array}{l}3 \\
6,3\end{array}\right\}$ & $\Omega_{1}=3, x \equiv y \equiv z \equiv t+1(\bmod 3)$ \\
\hline$\{6,3,6\}$ & $\Omega_{1}=0, x \equiv y(\bmod 3), \operatorname{gcd}(x, y, z, t)=1$ \\
\hline$\left\{\begin{array}{l}6 \\
3,6\end{array}\right\}$ & $\Omega_{1}=1, x \equiv y \equiv z+1 \equiv t+1(\bmod 3)$ \\
\hline
\end{tabular}

TABLE 2. Honeycombs using absolute $\Omega_{2}=0$, where $\Omega_{2}=t^{2}-3\left(x^{2}+y^{2}+z^{2}\right)$.

\begin{tabular}{|c|c|}
\hline Honeycomb & Equations and congruences \\
\hline$\{4,3,6\}$ & $\Omega_{2}=0$ \\
\hline$\left\{\begin{array}{l}4 \\
3,6\end{array}\right\}$ & $\Omega_{2}=3$ \\
\hline$\{6,3,4\}$ & $\Omega_{2}=1$ \\
\hline$\left\{\begin{array}{l}6 \\
3,4\end{array}\right\}$ & $\Omega_{2}=6$ \\
\hline
\end{tabular}


TABLE 3. Honeycombs using absolute $\Omega_{3}=0$, where $\Omega_{3}=t^{2}-\left(x^{2}+y^{2}+z^{2}\right)$.

\begin{tabular}{|c|c|}
\hline Honeycomb & Equations and congruences \\
\hline$\{3,4,4\}$ & $\Omega_{3}=0$ \\
\hline$\left\{\begin{array}{l}3 \\
4,4\end{array}\right\}$ & $\Omega_{3}=2$ \\
\hline$\{4,4,3\}$ & $\Omega_{3}=1$ \\
\hline$\left\{\begin{array}{l}4 \\
4,3\end{array}\right\}$ & $\Omega_{3}=6$ \\
\hline$\left\{\begin{array}{c}4,4 \\
3\end{array}\right\}$ & $\Omega_{3}=1, x \equiv y \equiv z \equiv t-1 \equiv 0(\bmod 2)$ \\
\hline$\{4,4,4\}$ & $\Omega_{3}=0, y+z \equiv 1(\bmod 2)$ \\
\hline
\end{tabular}

4. Bipartite graphs embedded in regular honeycombs. Joining the center of each face of $\{p, q, r\}$ to the midpoints of its $p$ edges, one obtains a bipartite graph with $p$-valent and $r$-valent vertices. Around every vertex of a cell of $\{p, q, r\}$ there are $q$ $p$-gons, so that the graph is of girth $2 q$ (Coxeter [1980, p. 309]). If $p=r=3$ the honeycombs are self-reciprocal, the corresponding graphs are trivalent and the two kinds of vertices behave alike. Any 3 consecutive edges of the graph belong to some $2 q$-gon, while 4 consecutive edges need not belong to any such polygon. This means that the graph is 3-regular (Tutte [1966, p. 62]).

When $q=3$, the regular spherical honeycomb $\{3,3,3\}$ (or 4-dimensional regular simplex) yields the Levi graph of a Desargues configuration (Coxeter [1980, p. 310]). $\{3,4,3\}$ is the 24-cell (Coxeter [1974, p. 32]), and the corresponding graph is Foster's 192A (Foster [1966, p. 21]). The edges of the graph can be 3-colored to provide the Cayley diagram for a group of order 192 with the following presentation:

$$
T_{1}^{2}=T_{2}^{2}=T_{3}^{2}=\left(T_{1} T_{2} T_{1} T_{3}\right)^{2}=\left(T_{2} T_{3} T_{2} T_{1}\right)^{2}=\left(T_{3} T_{1} T_{3} T_{2}\right)^{2}=1
$$

(Coxeter [1980, p. 320]).

The honeycombs $\{3, q, 3\}$ for $q>4$ yield infinite graphs. In the case $q=6$, as it will be shown, the graph can again be 3-colored in the manner which involves the 3 colors symmetrically.

5. An infinite graph of girth 12 . The symmetry group [3,6,3] of the honeycomb $\{3,6,3\}$ is generated by four reflections $R_{i}(i=1,2,3,4)$ with the presentation

(1) $\quad R_{i}^{2}=\left(R_{1} R_{2}\right)^{3}=\left(R_{2} R_{3}\right)^{6}=\left(R_{3} R_{4}\right)^{3}=1, \quad R_{3} \rightleftarrows R_{1} \rightleftarrows R_{4} \rightleftarrows R_{2}$.

Its "even" subgroup (of index 2) [3,6,3] ${ }^{+}$is generated by three rotations (products of pairs of four reflections)

$$
Q=R_{4} R_{3}, \quad R=R_{4} R_{1}, \quad S=R_{2} R_{1}
$$


satisfying the presentation

$$
Q^{3}=R^{2}=S^{3}=(Q R)^{2}=(R S)^{2}=(S R Q)^{6}=1 .
$$

That these relations suffice is easy to see by adjoining a new element $\dot{R}_{1}$ of order 2 which commutes with $R$ and $Q$, and transforms $S$ to $S^{-1}$.

As we saw already, reflections in the faces of the orthoscheme

$$
(1,0,0,0)(1,1,0,0)(1,1,1,0)(0,0,0,1)
$$

yield $[3,6,3]$ and the orbit of $(1,0,0,0)$ is $\{3,6,3\}$ inscribed in the absolute $\Omega_{1}=x y+x z+x t+y z+y t+z t=0$. In matrix notation

$$
\begin{aligned}
R_{1}=\left[\begin{array}{cccc}
0 & 1 & 0 & 0 \\
1 & 0 & 0 & 0 \\
0 & 0 & 1 & 0 \\
0 & 0 & 0 & 1
\end{array}\right], & R_{2}=\left[\begin{array}{cccc}
1 & 0 & 0 & 0 \\
0 & 0 & 1 & 0 \\
0 & 1 & 0 & 0 \\
0 & 0 & 0 & 1
\end{array}\right], \\
R_{3}=\left[\begin{array}{cccc}
1 & 0 & 0 & 0 \\
0 & 1 & 0 & 0 \\
1 & 1 & -1 & 1 \\
0 & 0 & 0 & 1
\end{array}\right], & R_{4}=\left[\begin{array}{cccc}
1 & 0 & 0 & 0 \\
0 & 1 & 0 & 0 \\
0 & 0 & 1 & 0 \\
1 & 1 & 1 & -1
\end{array}\right],
\end{aligned}
$$

$S$ and $Q$ are rotations through $2 \pi / 3$ about the lines $(0,0,0,1)(1,1,1,0)$ and $(1,0,0,0)(1,1,0,0)$, respectively, and $R$ is a half-turn about $(1,1,0,0)(1,1,1,0)$.

The trigonal rotations $Q$ and $S$ generate the subgroup $\left[3^{+}, 6,3^{+}\right]$of index 2 with the presentation

$$
Q^{3}=S^{3}=\left(Q^{-1} S^{-1} Q S\right)^{3}=1 .
$$

Adjoining an element $R$ of order 2 to (4) such that $R$ transforms $Q$ to $Q^{-1}$ and $S$ to $S^{-1}$ we get $[3,6,3]^{+}$with the presentation (3). The twist

$$
U=(Q S)^{-1}=R_{1} R_{2} R_{3} R_{4}=\left[\begin{array}{cccc}
2 & 2 & 0 & -1 \\
1 & 0 & 0 & 0 \\
0 & 1 & 0 & 0 \\
1 & 1 & 1 & -1
\end{array}\right]
$$

shifts a Petrie polygon of $\{3,6,3\}$ one step along itself (Coxeter [1973, p. 223]). In terms of $S$ and $U$ the presentation for $\left[3^{+}, 6,3^{+}\right]$is $S^{3}=(S U)^{3}=\left(S^{-1} U^{-1} S U\right)^{3}=1$. In this group, the three elements

$$
U_{1}=S^{-1} U S, \quad U_{2}=U, \quad U_{3}=S U S^{-1}
$$

generate a subgroup of index 3 with the presentation

$$
\left(U_{2}^{-1} U_{3}\right)^{3}=\left(U_{3}^{-1} U_{1}\right)^{3}=\left(U_{1}^{-1} U_{2}\right)^{3}=U_{1} U_{2} U_{3}=1 .
$$

Adjoining an element $S$ of order 3 which cyclically permutes $U_{1}, U_{2}$ and $U_{3}$, we get $\left[3^{+}, 6,3^{+}\right]$. In terms of $U_{1}$ and $U_{2}$ alone (6) becomes

$$
\left(U_{1} U_{2}^{2}\right)^{3}=\left(U_{1}^{2} U_{2}\right)^{3}=\left(U_{1}^{-1} U_{2}\right)^{3}=1 .
$$




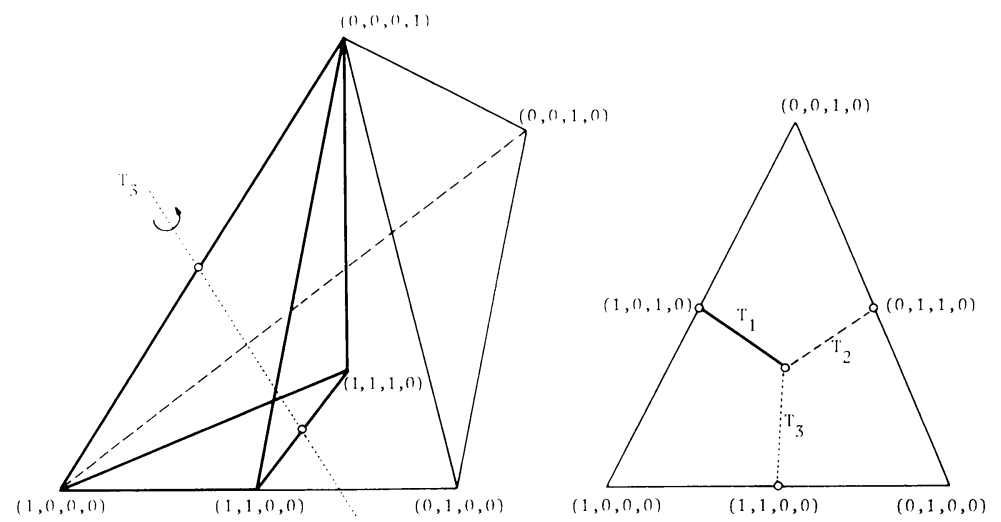

FIGURE 2

The half-turn

$$
T_{3}=\left[\begin{array}{cccc}
0 & 0 & 0 & 1 \\
1 & 1 & 1 & -1 \\
2 & 2 & -1 & 0 \\
3 & 0 & 0 & 0
\end{array}\right]
$$

reverses the opposite edges $(1,0,0,0)(0,0,0,1)$ and $(1,1,0,0)(1,1,1,0)$ of the orthoscheme for $\{3,6,3\}$ (Figure 2). $T_{3}$ interchanges $U_{1}$ with $U_{1}^{-1}$, and $U_{2}$ with $U_{2}^{-1}$. Adjoining $T_{3}$ to (7) we obtain a group with the presentation

$$
T_{3}^{2}=\left(U_{1} T_{3}\right)^{2}=\left(T_{3} U_{2}\right)^{2}=\left(U_{1} U_{2}^{2}\right)^{3}=\left(U_{1}^{2} U_{2}\right)^{3}=\left(U_{1}^{-1} U_{2}\right)^{3}=1 .
$$

In terms of $T_{1}=T_{3} U_{2}$ and $T_{2}=U_{1} T_{3}$ we have

$$
T_{1}^{2}=T_{2}^{2}=T_{3}^{2}=\left(T_{1} T_{2} T_{1} T_{3}\right)^{3}=\left(T_{2} T_{3} T_{2} T_{1}\right)^{3}=\left(T_{3} T_{1} T_{3} T_{2}\right)^{3}=1 .
$$

Since the rotation $S$ cyclically permutes $U_{1}=T_{2} T_{3}, U_{2}=T_{3} T_{1}$ and $U_{3}=T_{1} T_{2}$ it will do the same to $T_{1}, T_{2}$ and $T_{3}$. In fact, $T_{2}=S^{-1} T_{3} S$ is the half-turn reversing $(0,1,1,0)(1,1,1,0)$ and $(0,1,0,0)(0,0,0,1)$, while $T_{1}=S T_{3} S^{-1}$ is the half-turn reversing $(1,0,1,0)(1,1,1,0)$ and $(0,0,1,0)(0,0,0,1)$. In matrix notation,

$$
T_{2}=\left[\begin{array}{cccc}
-1 & 2 & 2 & 0 \\
0 & 0 & 0 & 1 \\
1 & 1 & 1 & -1 \\
0 & 3 & 0 & 0
\end{array}\right], \quad T_{1}=\left[\begin{array}{cccc}
1 & 1 & 1 & -1 \\
2 & -1 & 2 & 0 \\
0 & 0 & 0 & 1 \\
0 & 0 & 3 & 0
\end{array}\right] .
$$

A Cayley diagram for the group (8) can be drawn on the faces of $\{3,6,3\}$ by joining the centers of each face to the midpoints of its edges. For this purpose we represent the group elements $1, T_{1}, T_{2}, T_{3}$ by the points $(1,1,1,0),(1,0,1,0)$, $(0,1,1,0),(1,1,0,0)$. Then since $(1,0,1,0) T_{3}=(2,2,-1,1)$ we can identify this point with $T_{3} T_{1}$, and $(0,1,1,0) T_{3}=(3,3,0,-1)$ with $T_{3} T_{2}$ (Figure 3$)$, i.e. with the centers of the other two faces that radiate from the edge $(1,0,0,0)(0,1,0,0)$. Figure 4 shows the part of the diagram drawn on a cell $\{3,6\}$. The relations $\left(T_{1} T_{2} T_{1} T_{3}\right)^{3}=\left(T_{2} T_{3} T_{2} T_{1}\right)^{3}$ $=\left(T_{3} T_{1} T_{3} T_{2}\right)^{3}=1$ can be read off from the diagram in Figure 4 , as circuits surrounding the three vertices of a face. 


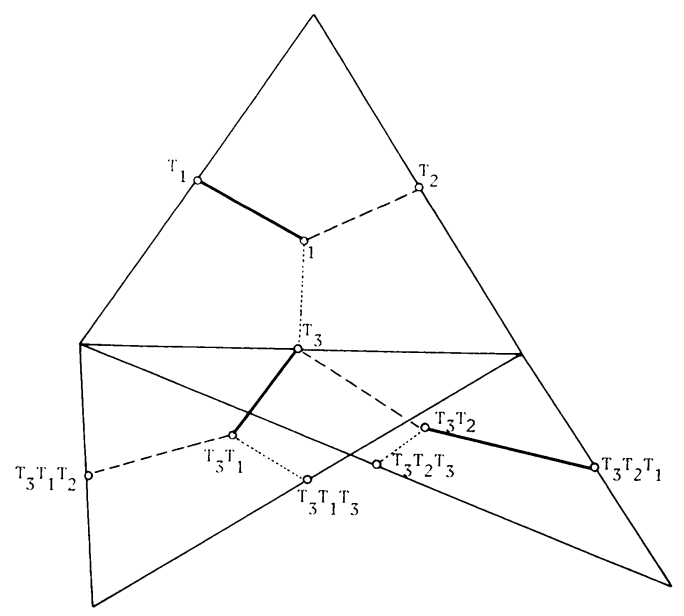

FIGURE 3

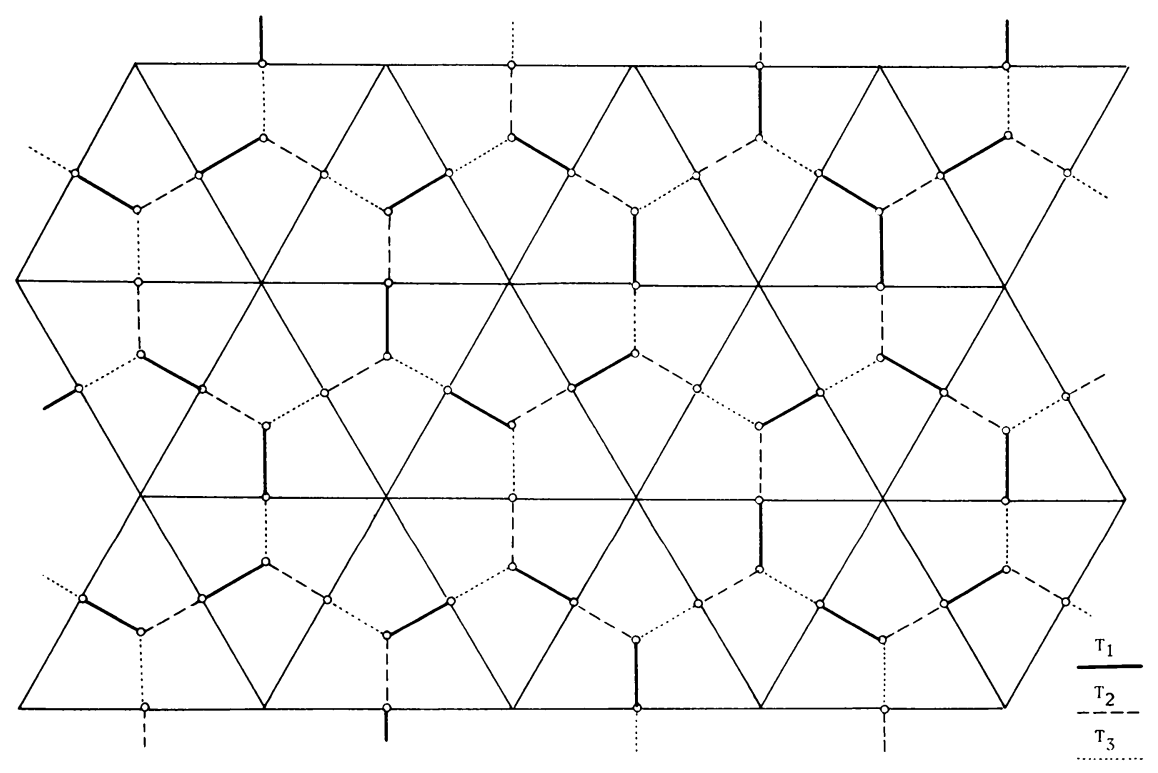

FIGURE 4

6. Some finite graphs of girth 12. By adjoining relations $\left(R_{1} R_{2} R_{3}\right)^{6}=\left(R_{4} R_{3} R_{2}\right)^{6}$ $=1$ to the infinite group (1) we obtain a finite group ${ }_{6}[3,6,3]_{6}$ which may be regarded as the symmetry group of a finite honeycomb ${ }_{6}\{3,6,3\}_{6}$. This honeycomb is thus seen to be derived from $\{3,6,3\}$ by identifying points separated by 6 steps along every Petrie polygon of the cell $\{3,6\}$ and vertex figure $\{6,3\}$. In other words, the finite honeycomb has cell $\{3,6\}_{6}$ and vertex figure $\{6,3\}_{6}$. 
In $_{6}[3,6,3]_{6}$, the even subgroup ${ }_{6}[3,6,3]_{6}^{+}$, generated by three rotations (2), has the presentation

$$
Q^{3}=R^{2}=S^{3}=(Q R)^{2}=(R S)^{2}=(S R Q)^{6}=\left(Q S Q S^{-1}\right)^{3}=\left(Q^{-1} S Q S\right)^{3}=1 .
$$

In this group $Q$ and $S$ generate the subgroup

$$
Q^{3}=S^{3}=\left(Q^{-1} S^{-1} Q S\right)^{3}=\left(Q S Q S^{-1}\right)^{3}=\left(Q^{-1} S Q S\right)^{3}=1
$$

of order $729=3^{6}$. This was checked by Coxeter who enumerated 27 cosets of a subgroup of order 27. The subgroup is the Burnside group $B_{2,3}$ (Coxeter and Moser [1980, p. 80]) generated by $S$ and $T=Q^{-1} S Q$ with the presentation

$$
S^{3}=T^{3}=(S T)^{3}=\left(S^{-1} T\right)^{3}=1 .
$$

The group ${ }_{6}[3,6,3]_{6}$ has order $4 \cdot 3^{6}$. Since the cells of ${ }_{6}\{3,6,3\}_{6}$ are $\{3,6\}_{6}$, $R_{1}, R_{2}$ and $R_{3}$ generate a subgroup of order $12 \cdot 3^{2}=108$ (Coxeter and Moser $\left[1980\right.$, p. 139]), and the honeycomb ${ }_{6}\{3,6,3\}_{6}$ has 27 toroidal cells. Its vertex figures $\{6,3\}_{6}$ are toroidal as well. Its cells have 18 triangular faces (Figure 5), giving $18 \cdot 27 / 2=243$ faces for the honeycomb. Being self-dual, the honeycomb has 27 vertices and 243 edges.

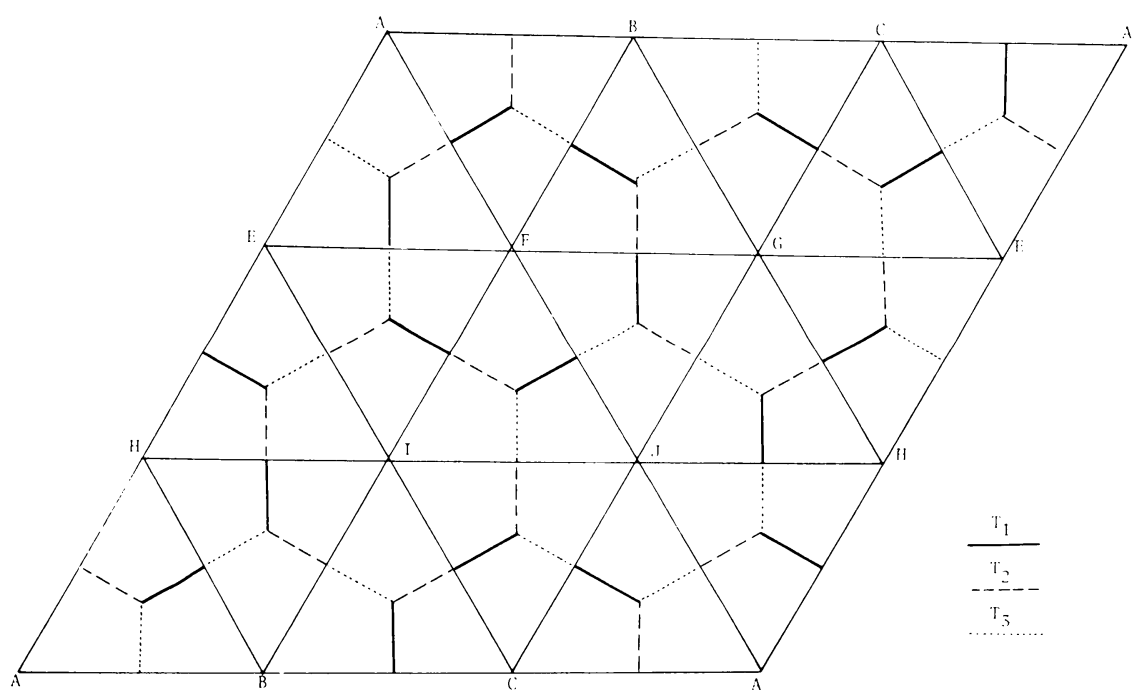

Figure 5

This reduces the infinite graph of girth 12 to a finite bipartite graph of girth 12 with $243+243=486$ vertices. This graph also can be 3 -colored involving three colors symmetrically. To see that, we find the subgroup of ${ }_{6}[3,6,3]_{6}$ of order $3^{5}$ generated by $U_{1}, U_{2}$ and $U_{3}$ are defined by (5), with the presentation

$$
\begin{aligned}
\left(U_{2}^{-1} U_{3}\right)^{3} & =\left(U_{3}^{-1} U_{1}\right)^{3}=\left(U_{1}^{-1} U_{2}\right)^{3}=U_{1} U_{2} U_{3} \\
& =U_{1}^{-1} U_{2} U_{3}^{-1} U_{1} U_{2}^{-1} U_{3}=U_{3}^{2} U_{2}^{2} U_{1}^{2}=1 .
\end{aligned}
$$


Introducing $T_{3}$, which transforms $U_{1}$ and $U_{2}$ into their respective inverses, so that $T_{1}=T_{3} U_{2}$ and $T_{2}=U_{1} T_{3}$ satisfy

$$
U_{1}=T_{2} T_{3}, \quad U_{2}=T_{3} T_{1}, \quad U_{3}=T_{1} T_{2},
$$

we obtain a group of order $2 \cdot 3^{5}=486$ :

$$
\begin{aligned}
T_{1}^{2} & =T_{2}^{2}=T_{3}^{2}=\left(T_{1} T_{2} T_{1} T_{3}\right)^{3}=\left(T_{2} T_{3} T_{2} T_{1}\right)^{3}=\left(T_{3} T_{1} T_{3} T_{2}\right)^{3} \\
& =T_{1} T_{2} T_{1} T_{3} T_{2} T_{3} T_{2} T_{1} T_{3} T_{1} T_{3} T_{2}=\left(T_{1} T_{2}\right)^{2}\left(T_{3} T_{1}\right)^{2}\left(T_{2} T_{3}\right)^{2}=1 .
\end{aligned}
$$

Its Cayley diagram is the above-mentioned "bipartite graph" whose automorphism group contains all the symmetries of the honeycomb plus an additional automorphism dualising the honeycomb and thus interchanging the midpoints of faces with the midpoints of edges. Hence the order of the group is $8 \cdot 3^{6}=2^{3} \cdot 486 \cdot 3 / 2$ making the graph 3-regular.

The addition of the relations

$$
\left(R_{3} R_{4} R_{1} R_{2}\right)^{3}\left(R_{4} R_{3} R_{2} R_{1}\right)^{3}=\left(R_{3} R_{4} R_{2} R_{1}\right)^{3}\left(R_{4} R_{3} R_{1} R_{2}\right)^{3}=1
$$

to ${ }_{6}[3,6,3]_{6}$ gives us a group whose order $108 \cdot 9=972$ can be found by enumerating the 9 cosets of the subgroup $[3,6]_{6}$ generated by $R_{1}, R_{2}$ and $R_{3}$. Hence the new honeycomb has 9 toroidal cells $[3,6]_{6}, 18 \cdot 9 / 2=81$ faces, 81 edges and 9 vertices. It was found independently by L. Danzer, who remarked that it was degenerated in various ways.

The additional relations (10) in terms of $U_{1}, U_{2}$ and $U_{3}$ can be written as

$$
U_{3}^{3} U_{2}^{-3}=U_{3} U_{2} U_{1} U_{2}^{-1} U_{3}^{-1} U_{1}^{-1}=1,
$$

or in terms of $T_{1}, T_{2}$ and $T_{3}$,

$$
\left(T_{1} T_{2}\right)^{3}\left(T_{1} T_{3}\right)^{3}=\left(T_{1} T_{2} T_{3}\right)^{2}\left(T_{1} T_{3} T_{2}\right)^{2}=1 .
$$

The addition of these relations to (9) shows the " $T$ " group to be of order 162 . Its Cayley diagram is Foster's $162 \mathrm{C}$ (Foster [1966, p. 21]), which is 3-regular and of girth 12. This is, of course, the same graph as the bipartite graph obtained from the above polytope.

7. A regular honeycomb with five toroidal cells. Let ${ }_{4}\{3,6,3\}_{4}$ denote the honeycomb obtained from $\{3,6,3\}$ after identifying points separated by four steps along every Petrie polygon of a cell, as well as of a vertex figure. With the addition of relations $\left(R_{1} R_{2} R_{3}\right)^{4}=\left(R_{4} R_{3} R_{2}\right)^{4}=1$ to (1) we obtain the finite group ${ }_{4}[3,6,3]_{4}$. In this group $R_{1}, R_{2}$ and $R_{3}$ generated the subgroup $[3,6]_{4} \cong G^{3,6,4} \cong C_{2} \times S_{4}$ (Coxeter and Moser [1980, p. 139]) of order 48, and index 5, making the whole group of order 240 . Hence, ${ }_{4}\{3,6,3\}_{4}$ has toroidal cells $\{3,6\}_{4}$, and being self-dual, 5 vertices. Each cell has 8 triangles, so that the whole honeycomb has $8 \cdot 5 / 2=20$ triangles, and likewise 20 edges. Every two cells have two common faces. tion

The generators of ${ }_{4}[3,6,3]_{4}$, as permutations of degree seven, have the representa-

$$
R_{1}=(12)(67), \quad R_{2}=(23)(67), \quad R_{3}=(34), \quad R_{4}=(45) .
$$


Since $\left(R_{2} R_{3}\right)^{3}=(67)$ generates $C_{2}$ and commutes with all elements of the $S_{5}$ generated by (12), (23), (34) and (45), it follows that the group of order 240 is $C_{2} \times S_{5}$.

The bipartite graph corresponding to this honeycomb has 40 vertices. By identifying the opposite faces and edges of ${ }_{4}\{3,6,3\}_{4}$ we obtain $\{3,3,3\}$, so that the graph on 40 vertices collapses to the Levi graph of a Desargues configuration. The graph of 40 vertices has a Hamiltonian circuit, and in Frucht's "antipalindromic" notation (Coxeter, Frucht and Powers [1981, p. 12]) can be described as $[15,9,-9,-15]^{10}$.

The graph with 40 vertices is 3 -regular and it has $40 \cdot 3 / 2=60$ edges, so that the group of automorphisms has $60 \cdot 2^{3}=480$ elements. It contains all the symmetries of the honeycomb, plus an additional automorphism dualising the honeycomb and thus interchanging the midpoints of faces with the midpoints of edges. The corresponding " $T$ " group is $C_{2} \times A_{5}$. Its Cayley diagram is Foster's 120B, providing 3-fold covering for the graph on 40 vertices.

8. A regular honeycomb with forty toroidal cells. Let us consider trigonal rotations $S$ and $Q$ generating $\left[3^{+}, 6,3^{+}\right]$with the representation

$$
S=\left[\begin{array}{cc}
0 & 1 \\
-1 & -1
\end{array}\right] \text { and } Q=\left[\begin{array}{cc}
\omega^{2} & 0 \\
0 & \omega
\end{array}\right]
$$

where $\omega$, being a cube root of unity, satisfies the equation $\omega^{2}+\omega+1=0 . S$ and $Q$ have determinants equal to 1 , and hence generate a subgroup of $\operatorname{SL}(2, \mathbf{Z}[\omega])$. Define $V=Q S^{-1} Q S$ and $W=Q^{-1} S^{-1} Q^{-1} S$; then

$$
V=\left[\begin{array}{cc}
1 & 0 \\
\omega-1 & 1
\end{array}\right], \quad W=\left[\begin{array}{cc}
1 & 0 \\
\omega^{2}-1 & 1
\end{array}\right]
$$

and thus

$$
V W=\left[\begin{array}{cc}
1 & 0 \\
-3 & 1
\end{array}\right], \quad V^{2} W=\left[\begin{array}{cc}
1 & 0 \\
\omega-4 & 1
\end{array}\right], \quad S V W=\left[\begin{array}{cc}
0 & 1 \\
-1 & -4
\end{array}\right]
$$

When entries of the matrices in $\operatorname{SL}(2, \mathbf{Z}[\omega])$ are reduced $\bmod 4$, we obtain $\operatorname{SL}\left(2, \mathbf{Z}_{4}[\omega]\right)$. Then (11) becomes

$$
V W=\left[\begin{array}{ll}
1 & 0 \\
1 & 1
\end{array}\right], \quad V^{2} W=\left[\begin{array}{ll}
1 & 0 \\
\omega & 1
\end{array}\right], \quad S V W=\left[\begin{array}{cc}
0 & -1 \\
-1 & 0
\end{array}\right] .
$$

But $\operatorname{SL}(2, \mathbf{Z}[\omega])$ is generated by

$$
\left[\begin{array}{cc}
\omega^{2} & 0 \\
0 & \omega
\end{array}\right], \quad\left[\begin{array}{ll}
1 & 0 \\
1 & 1
\end{array}\right], \quad\left[\begin{array}{ll}
1 & 0 \\
\omega & 1
\end{array}\right] \text { and }\left[\begin{array}{cc}
0 & 1 \\
-1 & 0
\end{array}\right]
$$

(Bianchi [1891, p. 314]) and hence $S$ and $Q$ generate $\operatorname{SL}\left(2, \mathbf{Z}_{4}[\omega]\right){ }^{\prime}$

$S$ and $Q$ generate $\operatorname{PSL}\left(2, \mathbf{Z}_{4}[\omega]\right)$ of order 1920 , with the presentation

$$
Q^{3}=S^{3}=(Q S)^{5}=\left(Q^{-1} S^{-1} Q S\right)^{3}=(S Q S Q S)^{4}=(Q S Q S Q)^{4}=1 .
$$

Sufficiency of this presentation was found by A. Sinkov, using a computer for the coset enumeration. To this group we adjoin $R$, such that $R^{2}=1, R Q R=Q^{-1}$ and

\footnotetext{
${ }^{1}$ This was kindly supplied by P. L. Waterman.
} 
$R S R=S^{-1}$, to obtain

$$
\begin{aligned}
Q^{3} & =R^{2}=S^{3}=(Q S)^{5}=(Q R)^{2}=(S R)^{2}=(R Q S)^{6} \\
& =(S Q S Q S)^{4}=(Q S Q S Q)^{4}=1
\end{aligned}
$$

Adjoining an element $R_{1}$, such that $R_{1}^{2}=1, R_{1} Q R_{1}=Q, R_{1} R R_{1}=R$ and $R_{1} S R_{1}=$ $S^{-1}$ we obtain a yet larger group of order 7680. The group generated by $R_{1}, R_{2}=S R_{1}$, $R_{3}=R_{1} R Q$ and $R_{4}=R_{1} R$ has the presentation

$$
\begin{gathered}
R_{i}^{2}=\left(R_{1} R_{2}\right)^{3}=\left(R_{2} R_{3}\right)^{6}=\left(R_{3} R_{4}\right)^{3}=\left(R_{1} R_{2} R_{3}\right)^{8} \\
=\left(R_{1} R_{2} R_{3} R_{4}\right)^{5}=\left(R_{2} R_{3} R_{4}\right)^{8}=1, \\
R_{3} \rightleftarrows R_{1} \rightleftarrows R_{4} \rightleftarrows R_{2} .
\end{gathered}
$$

In this group $R_{1}, R_{2}$ and $R_{3}$ generate a subgroup of index 40. Since $R_{1} R_{2} R_{3}$ is of order 8 , this group is $[3,6]_{8} \cong G^{3,6,8}$, of order 192 (Coxeter and Moser [1980, p. 112]).

From $\{3,6,3\}$ we obtain a finite honeycomb the following way. First we identify points separated by 8 steps along Petrie polygons of cells and points separated by 8 steps along Petrie polygons of vertex figures. But this is not enough to make the honeycombs finite since the addition of $\left(R_{1} R_{2} R_{3}\right)^{8}=\left(R_{2} R_{3} R_{4}\right)^{8}=1$ to (1) does not give a finite group. Next we identify all points separated by 5 steps along Petrie polygons of the honeycomb to obtain a finite honeycomb with the symmetry group (12). The honeycomb has 40 cells and, being self-dual, 40 vertices. Each cell has 48 edges and each edge is shared by 3 cells, giving $40 \cdot 48 / 3=640$ edges for the honeycomb. Self-duality implies that the honeycomb has 640 faces.

The trivalent bipartite graph corresponding to this honeycomb has $640+640=$ 1280 vertices and $1280 \cdot 3 / 2=1920$ edges. It is 3 -regular since 4 consecutive edges belong either to a decagon (when drawn on those faces which belong to one of the 5 -sided Petrie polygons of the polytope) or to a dodecagon (when drawn on a cell around one of its vertices). The " $T$ " group for this honeycomb is of order 3840 , providing a 3-fold covering for the bipartite graph.

\section{REFERENCES}

[1891] L. Bianchi, Geometrische Darstellung der Gruppen linearer Substitutionen mit ganzen complexen Coefficienten nebst Anwendungen auf die Zahlentheorie, Math. Ann. 38, 313-333.

[1968] N. Bourbaki, Groupes et algèbres de Lie, Chapitres 4, 5 et 6, Hermann, Paris.

[1939] H. S. M. Coxeter, The abstract groups $G^{m, n . p}$, Trans. Amer. Math. Soc. 45, 73-150.

[1968] __ Twelve geometric essays, Southern Illinois Univ. Press, Carbondale, Ill.

[1973] __, Regular polytopes, 3rd ed., Dover, New York.

[1974] __ Regular complex polytopes, Cambridge Univ. Press, London and New York.

[1980] _. The edges and faces of a 4-dimensional polytope, Congress. Numer. 28, 309-334.

[1981] H. S. M. Coxeter, R. Frucht and D. L. Powers, Zero-symmetric graphs: Trivalent graphical regular representations of groups, Academic Press, New York.

[1980] H. S. M. Coxeter and W. O. J. Moser, Generators and relations for discrete groups, 4th ed., Springer, Berlin.

[1950] H. S. M. Coxeter and G. T. Whitrow, World structure and non-Euclidean honeycombs, Proc. Roy. Soc. London Ser. A 201, 417-437.

[1966] R. M. Foster, A census of trivalent symmetrical graphs. I, presented at the Conference on Graph Theory and Combinatorial Analysis, University of Waterloo, Ontario. 
[1964] C. W. L. Garner, Polyhedra and honeycombs in hyperbolic space, Ph.D. Thesis, University of Toronto.

[1976] B. Grünbaum, Regularity of graphs, complexes and designs, Colloq. Internat. CNRS, Problèmes Combinatoires et Théorie des Graphes, Orsay.

[1929] D. M. Y. Sommerville, An introduction to the geometry of $n$ dimensions, Methuen, London.

[1966] W. T. Tutte, Connectivity in graphs, Univ. of Toronto Press.

Department of Mathematics, University of Saskatchewan, Saskatoon, Saskatchewan S7N OW0, CANADA

Current address: Department of Mathematics, University of Toronto, Toronto, Ontario, Canada M5S $1 \mathrm{Al}$ 